\title{
TWO THEOREMS OF EULER AND A GENERAL PARTITION THEOREM
}

\author{
GEORGE E. ANDREWS ${ }^{1}$
}

1. Introduction. Euler proved that the partitions of a natural number $n$ into distinct parts are equinumerous with the partitions of $n$ into odd parts [2, p. 277]. A second theorem due to Euler states that every natural number is uniquely representable as a sum of distinct powers of $2[2$, p. 277].

If $S_{1}$ and $S_{2}$ are subsets of the natural numbers $N$, let us call $\left(S_{1}, S_{2}\right)$ an Euler-pair if for all natural numbers, $n$, the number of partitions of $n$ into distinct parts taken from $S_{1}$ equals the number of partitions of $n$ into parts taken from $S_{2}$. Euler's first theorem may then be stated by saying $N$ and $\{n \in N \mid 2 \nmid n\}$ are an Euler-pair, and his second theorem may be stated by saying that $\left\{2^{n} \mid n \in N\right.$ or $\left.n=0\right\}$ and $\{1\}$ are an Euler-pair. Other examples of Euler-pairs are

$$
(\{n \in N \mid 3 \nmid n\},\{n \in N \mid n \equiv 1,5(\bmod 6)\})
$$

due to I. J. Schur [3, p. 495], and

$$
(\{n \in N \mid n \equiv 2,4,5(\bmod 6)\},\{n \in N \mid n \equiv 2,5,11(\bmod 12)\})
$$

due to $\mathrm{H}$. Göllnitz [1, p. 175]. The object of this paper is to give a simple characterization of Euler-pairs.

Throughout this paper all sets $S_{\boldsymbol{i}}$ which we consider will be subsets of the natural numbers $N$. The notation $m S_{i}$ denotes the set $\left\{m n \mid n \in S_{i}\right\}$. The notation $S_{i}-S_{j}$ denotes the set $\left\{n \in S_{i} \mid n \in S_{j}\right\}$. By $p\left(S_{i} ; n\right)$ we denote the number of partitions of $n$ into parts taken from $S_{i}$. By $q\left(S_{i} ; n\right)$ we denote the number of partitions of $n$ into distinct parts taken from $S_{i}$. We shall write $S_{i}=\left\{s_{1}(i), s_{2}(i), s_{3}(i), \cdots\right\}$ where the elements are arranged in ascending order of magnitude.

THEOREM 1. $\left(S_{1}, S_{2}\right)$ is an Euler pair if and only if $2 S_{1} \subseteq S_{1}$ and $S_{2}=S_{1}-2 S_{1}$.

In $\$ 2$ we shall prove Theorem 1. In $\$ 3$ we examine some of the corollaries of Theorem 1.

2. Proof of Theorem 1. First we require the following result.

Lemma. If for every natural number $n, p\left(S_{1} ; n\right)=p\left(S_{2} ; n\right)$, then

Received by the editors November 14, 1967.

${ }^{1}$ Partially supported by National Science Foundation Grant GP-6663. 
$S_{1}=S_{2}$. Indeed if $m$ is the least integer in $\left(S_{1} \cup S_{2}\right)-\left(S_{1} \cap S_{2}\right)$, then $p\left(S_{1} ; m\right) \neq p\left(S_{2} ; m\right)$.

Proof. We need only show that the second statement is valid. Without loss of generality we assume $m=s_{r}(1)$. Now $p\left(S_{1} ; m\right)$ is just the number of partitions of $m$ into parts taken from the set $\left\{s_{1}(1)\right.$, $\left.s_{2}(1), \cdots, s_{r}(1)\right\}$. On the other hand, $p\left(S_{2} ; m\right)$ is just the number of partitions of $m$ into parts taken from $S_{2}$ which do not exceed $m$ in magnitude; consequently by the definition of $m, p\left(S_{2} ; m\right)$ is just the number of partitions of $m$ into parts taken from the set $\left\{s_{1}(1), s_{2}(1)\right.$, $\left.\cdots, s_{r-1}(1)\right\}$. Therefore $p\left(S_{1} ; m\right)=p\left(S_{2} ; m\right)+1$. This completes the proof of the lemma.

Proof of Theorem 1. First we treat sufficiency. The generating function for $q\left(S_{1} ; n\right)$ is $\prod_{n \in S_{1}}\left(1+q^{n}\right)$, which is absolutely and uniformly convergent for $|q| \leqq 1-\delta$.

The generating function for $p\left(S_{2} ; n\right)$ is $\prod_{n \in S_{2}}\left(1-q^{n}\right)^{-1}$, again absolutely and uniformly convergent for $|q| \leqq 1-\delta$.

Now assuming $2 S_{1} \subseteq S_{1}$ and $S_{2}=S_{1}-2 S_{1}$, we have

$$
\begin{aligned}
\prod_{n \in S_{1}}\left(1+q^{n}\right) & =\prod_{n \in S_{1}}\left(1-q^{2 n}\right)\left(1-q^{n}\right)^{-1} \\
& =\prod_{n \in S_{1}-2 S_{1}}\left(1-q^{n}\right)^{-1}=\prod_{n \in S_{2}}\left(1-q^{n}\right)^{-1} .
\end{aligned}
$$

This establishes that $q\left(S_{1} ; n\right)=p\left(S_{2} ; n\right)$ for all $n$. Therefore $\left(S_{1}, S_{2}\right)$ is an Euler-pair.

Next we treat necessity. We suppose that $\left(S_{1}, S_{2}\right)$ is an Euler-pair. If $\left(S_{1}, S_{3}\right)$ were also an Euler-pair, then by the lemma and the definition of Euler-pair we have $S_{2}=S_{3}$. If we can show $2 S_{1} \subseteq S_{1}$, then as above we know that $\left(S_{1}, S_{1}-2 S_{1}\right)$ is an Euler-pair and hence $S_{2}$ $=S_{1}-2 S_{1}$. Thus we need only show that $2 S_{1} \subseteq S_{1}$.

Suppose $2 S_{1} \nsubseteq S_{1}$. Let $s_{r}(1)$ be the least element of $S_{1}$ such that $2 s_{r}(1) \notin S_{1}$. Now

$$
\begin{aligned}
\prod_{n \in S_{2} ; n<2 s_{r}(1)} & \left(1-q^{n}\right)^{-1} \prod_{n \in S_{2 ;} ; n \geq 2 s_{r}(1)}\left(1-q^{n}\right)^{-1} \\
= & \prod_{n \in S_{1}}\left(1+q^{n}\right)=\prod_{n \in S_{1}}\left(1-q^{2 n}\right)\left(1-q^{n}\right)^{-1} \\
= & \prod_{n \in S_{1} ; n<s_{r}(1)}\left(1-q^{2 n}\right) \prod_{n \in S_{1 ;} ; n<2 s_{r}(1)}\left(1-q^{n}\right)^{-1} \prod_{n \in S_{1} ; n \geq s_{r}(1)}\left(1-q^{2 n}\right) \\
& \cdot \prod_{n \in S_{1} ; n>2 s_{r}(1)}\left(1-q^{n}\right)^{-1}=\prod_{n \in S_{1}-2 S_{1} ; n<2 s_{r}(1)}\left(1-q^{n}\right)^{-1} \\
& \cdot \prod_{n \in S_{1} ; n \geq \varepsilon_{r}(1)}\left(1-q^{2 n}\right) \prod_{n \in S_{1} ; n>2 s_{r}(1)}\left(1-q^{n}\right)^{-1} .
\end{aligned}
$$


Thus from the above identity we see that

$$
\prod_{n \in S_{2} ; n<2 \varepsilon_{r}(1)}\left(1-q^{n}\right)^{-1} \text {. and } \prod_{n \in S_{1}-2 S_{1} ; n<2 \varepsilon_{r}(1)}\left(1-q^{n}\right)^{-1}
$$

agree as power series in $q$ for the first $2 s_{r}(1)$ coefficients. However, if these two functions were unequal, then by the lemma they would have unequal coefficients among the first $2 s_{r}(1)$ coefficients; hence these products are equal. Thus, cancelling them in the above equation, we obtain

$$
\prod_{n \in S_{2 ; n \geq 2 s_{r}(1)}}\left(1-q^{n}\right)^{-1}=\prod_{n \in S_{1} ; n \geq s_{r}(1)}\left(1-q^{2 n}\right) \prod_{n \in S_{2 ; n>2 s_{r}(1)}}\left(1-q^{n}\right)^{-1} .
$$

Consider now the coefficient of $q^{2 s_{r}(1)}$ in the power series expansion of both sides of this equation. On the left-hand side it is either 0 or 1 , and on the right-hand side it is -1 . Thus we have a contradiction, and therefore we must have $2 S_{1} \subseteq S_{1}$. This completes the proof of Theorem 1.

3. Corollaries. We start with the following special case of Theorem 1.

Theorem 2. Let $S_{1} \subseteq N$ be such that $n \in S_{1}$ if and only if $2 n \in S_{1}$. Let $S_{2}=\left\{n \in S_{1} \mid n \equiv 1(\bmod 2)\right\}$. Then $\left(S_{1}, S_{2}\right)$ is an Euler-pair.

Proof. We need only show

$$
S_{1}-2 S_{1}=\left\{n \in S_{1} \mid n \equiv 1(\bmod 2)\right\} \text {. }
$$

Clearly the set on the left-hand side contains the set on the right-hand side. On the other hand, if $n \in S_{1}$ and $n=2 m$, then by hypothesis $m \in S_{1}$, hence $n \in 2 S_{1}$; consequently $n \notin S_{1}-2 S_{1}$. Thus we have Theorem 2.

Both of Euler's theorems are obvious consequences of Theorem 2, as is Schur's result. Göllnitz's theorem is not a corollary of Theorem 2 , but it is easily deduced from Theorem 1 . We may also prove many other partition theorems which do not seem to have been noted.

THEOREM 3. The number of partitions of a natural number $n$ into distinct parts, each of which is representable as the sum of two squares, equals the number of partitions of $n$ into odd parts each of which is representable as the sum of two squares.

Proof. By [2, p. 299], $n$ is representable as the sum of two squares if and only if $2 b$ is. The desired result now follows from Theorem 2.

THEOREM 4. If $p$ is a prime $\equiv 1,7(\bmod 8)$, then the number of partitions of a number $n$ into distinct quadratic residues $(\bmod p)$ equals the 
number of partitions of $n$ into odd parts which are quadratic residues $(\bmod p)$.

Proof. Since $p \equiv 1,7(\bmod 8), 2$ is a quadratic residue $(\bmod p)$ $[2$, p. 75$]$. Thus $n$ is a quadratic residue $(\bmod p)$ if and only if $2 n$ is a quadratic residue $(\bmod p)$. The desired result now follows from Theorem 2.

\section{REFERENCES}

1. H. Göllnitz, Partitionen mit Differenzenbedingungen, J. Reine Angew. Math. 225 (1967), 154-190.

2. G. H. Hardy and E. M. Wright, An introduction to the theory of numbers, 4th ed., Oxford Univ. Press, London, 1960.

3. I. J. Schur, Zur additiven Zahlentheorie, S.-B. Akad. Wiss. Berlin (1926), 488495.

Pennsylvania State University 